\title{
Predictors of body mass index in female parents whose children participate in a competitive, creative, problem-solving program
}

\author{
Naima Moustaid-Moussa 1,2,3*, Carol A. Costello ${ }^{4}$, \\ Betty P. Greer ${ }^{3}$, Marsha Spence ${ }^{5}$, Eugene Fitzhugh ${ }^{6}$, \\ Robert Muenchen ${ }^{7}$ and Nishan S. Kalupahana ${ }^{8}$
}

\begin{abstract}
'UT Obesity Research Center, The University of Tennessee, Knoxville, Tennessee, USA; ${ }^{2}$ Department of Animal Science, The University of Tennessee, Knoxville, Tennessee, USA; ${ }^{3}$ The University of Tennessee Extension, Family and Consumer Sciences, The University of Tennessee, Knoxville, Tennessee, USA; ${ }^{4}$ Department of Retail, Hospitality and Tourism, The University of Tennessee, Knoxville, Tennessee, USA; ${ }^{5}$ Department of Nutrition, The University of Tennessee, Knoxville, Tennessee, USA; 'Department of Kinesiology, The University of Tennessee, Knoxville, Tennessee, USA; ${ }^{7}$ Office of Information Technology, The University of Tennessee, Knoxville, Tennessee, USA; ${ }^{8}$ University of Peradeniya, Sri Lanka
\end{abstract}

Abstract

Background: Recent findings from our research indicate that children participating in a creative afterschool program exhibit overall healthier lifestyle practices compared to the average US pediatric population. This observation led us to investigate the prevalence of overweight/obesity and lifestyle practices of their parents. Objective: To determine the strongest predictors of weight status for female parents whose children were participating in such creative afterschool program.

Design: Surveyed subjects were parents of children who competed in the 2008 and 2009 Destination ImagiNation ${ }^{\circledR}$ Global Finals in Knoxville, Tennessee. A total of 4,608 children participated in data collection, with parental consent. For the combined 2 years, 1,118 parents, $87 \%$ of whom were females $(n=1,032)$ completed online questionnaires, which were based on the Behavioral Risk Factor Surveillance System and included self-reported height, weight, dietary intake, physical activity, and socioeconomic status. The majority of this population was white, and less than 5\% were African American or Hispanic.

Results: We report here results obtained for the female parents. Only $45.2 \%$ of these female parents were overweight/obese, compared to a national average of $64.1 \%$ reported by the National Health Nutrition Examination Surveys for 2007-2008. Furthermore, this population was significantly more physically active compared to national average. Most parents $(76 \%)$ had completed a college degree and reported high incomes. Parents with the lowest income were the most obese in this population. Finally, we found a significant association between parent and child weight status.

Conclusions: These studies demonstrate that female parents of children who have healthy lifestyles were physically active, which likely accounts for the parents' lower overweight/obesity rates. In addition to physical activity, income and percentage of calories from fat were all predictors of weight status.

Keywords: physical activity; diet; health behaviors; parents; obesity; overweight; BRFSS

Received: I March 2012; Revised: 7 July 2012; Accepted: 14 July 2012; Published: 16 August 2012

$\mathrm{O}$ verweight and obesity are due to multifactorial, complex interactions between genetics, dietary intake, physical activity, and the social, physical, and nutritional environments (1). Although addressing all of these aspects is important for preventing and treating overweight and obesity, understanding which of these components most likely predict overweight and obesity is essential in developing effective overweight and obesity prevention and treatment programs. 


\section{Obesity and overweight prevalence and relationship to poor health}

The obesity rate in the United States has reached an epidemic level, with approximately one-third of the adult population classified as obese [body mass index $(\mathrm{BMI}) \geq 30$ ]. According to the National Health Nutrition Examination Surveys (NHANES), $64.1 \%$ of women were overweight or obese between the years 2007 and 2008 (2). Differences in overweight and obesity prevalence exist among adults of various ethnic and racial backgrounds (2). In addition, certain sociodemographic factors such as income/socioeconomic status (SES) and education may contribute to overall weight status (3). Overweight and obesity are concerning conditions because numerous studies have suggested a strong correlation between obesity and the onset of chronic diseases such as cardiovascular disease and type 2 diabetes (4).

The Centers for Disease Control and Prevention estimate that $17 \%$ of youth aged 2-19 years are obese (5). Research also suggests that parents are children's immediate role models and can impact their children's lifestyle, including food consumption and physical activity (6).

\section{Dietary consumption, physical activity, and obesity}

According to the World Health Organization (WHO), obesity is a concerning global epidemic that is growing at an exponential rate. WHO states that the potential cause of obesity is consumption of foods that are high in energy density, are low in nutrient density, and lack vitamins, minerals, and macronutrients (7), combined with sedentary lives. Obesity is caused by an energy imbalance resulting from excessive caloric intake and low energy expenditure, as discussed below. Thus far, all research indicates that women who consume diets that are high in fat and low in fiber tend to be the most vulnerable to development of overweight or obese weight status. The overall recommendation for weight control is to consume diets that are rich in fiber, fruits, and vegetables and low in fat, especially saturated and trans fat, while engaging in regular physical activity (7).

According to the 2008 physical activity guidelines for Americans, adults between the ages of 18 and 64 years should get at least 150 min of moderate-intensity physical activity per week to maintain good health or $300 \mathrm{~min}$ of moderate-to-vigorous physical activity (MVPA) for weight loss (8). Physical activity has been suggested to help lower hypertension, type 2 diabetes, heart disease, and metabolic syndrome. Evidence also suggests that physical activity combined with a well-balanced diet is the most effective obesity preventative method $(9,10)$.

\section{Children's fitness and parental influence}

Studies have shown that parental modeling is an important factor in determining the level of physical activity and weight status in children (11). Indeed, a greater level of MVPA is seen in children whose parents also have a higher level of MVPA (12). Additional studies further suggest the influence of parenting style on children's objectively measured physical activity (13). Conversely, the presence of sedentary behaviors such as television time also correlates well between parents and their children (14). In line with these findings, Hills et al. proposed that one productive approach to childhood obesity would be to support parents, teachers, and healthcare workers to encourage children to be physically active (15).

Since children have been shown to be influenced by parents' modeling of physical activity and sedentary behavior and by encouragement of healthy eating in all socioeconomic conditions, it is important to identify the parental behaviors of children who are fit. We hypothesized that parents of children who exhibit healthy behaviors and lower BMIs than the average US pediatric population are characterized by lower rates of obesity, higher physical activity, and lower energy intakes. To test this hypothesis, we determined the strongest predictors of weight status of female parents whose children are fit. More specifically, we determined (1) what parental behaviors are predicted to be associated with parents' overweight and obesity, (2) any relationship between parents' and children's BMI, and (3) what is different about parents whose children exhibit healthy behavior. We studied mothers of fit children who are enrolled in a creative afterschool program to examine whether these mothers were behaviorally and anthropometrically different from the rest of the nation.

\section{Methods}

The study reported here was part of a larger project investigating health behaviors and practices of adolescents $(n=4,608)$ who participated in the Destination ImagiNation ${ }^{\circledR}$ (DI) Global Finals held in Knoxville, TN, in May 2008 and 2009. Students' weight, height, and survey responses were collected onsite.

The current study surveyed a total of 1,188 parents, of which 1,032 were female parents/guardians of this adolescent population. Self-reported survey and anthropometric data were collected from a sample of parents who gave consent for their children to participate in the student study. Parents received a letter and code number 1 week after their children competed in the DI Global Finals. The letter thanked the parents for allowing their children to participate in the study and requested parents' participation in a survey, either online or via mail. The letter contained the URL for the online survey, informed consent for parents' participation, and the code number, which linked parents' data to their children's data. We report here results obtained for the female parents, who were $87 \%$ of parents responding. 
Surveys consisted of dietary, physical activity, and weight perception/management questions from the 2007 Behavioral Risk Factor Surveillance System (BRFSS) (16), along with (1) the adult version of the Macarthur Scale of Subjective Social Status and (2) the Rapid Food Screener (17) (conducted on a subset of the participants), which was developed at the University of California, Berkeley, CA. Specifically, the survey collected the following data: (1) how often respondents consumed fruit juice, fruit, green salad, potatoes (not including French fries, fried potatoes, or potato chips), carrots, and other vegetables; (2) an inventory of foods present in the home (various categories of milk/dairy, cheese, salad dressing, cereal, bread/pasta/rice, baked goods, fresh vegetables, frozen vegetables, fresh fruit, meat/sausage, crackers/chips, and legumes); (3) grocery-shopping habits; (4) frequency of eating away from home; (5) amount and frequency of moderate or vigorous physical activity; (6) standing in society in relation to money, education, and employment; (7) standing in the community; (8) assessment of weight (underweight/overweight, change in weight, and efforts to change weight); (9) relationship to DI participant; (10) age; (11) race; (12) marital status; (13) education; (14) income; and (15) height and weight.

Data analyses included descriptive statistics, chisquared test for cross-tabulation of weight status between

Table 1. Female parents' demographic characteristics and descriptive statistics $(n=1,032)$

\begin{tabular}{|c|c|c|}
\hline & Frequency $(n)$ & $\%$ \\
\hline \multicolumn{3}{|l|}{ Race/ethnicity } \\
\hline White & 989 & 95.8 \\
\hline Other & 43 & 4.2 \\
\hline \multicolumn{3}{|c|}{ Annual household income } \\
\hline$<\$ 25,000$ & 16 & 1.6 \\
\hline$\$ 25,000-\$ 50,000$ & 80 & 7.8 \\
\hline$\$ 50,00 \mathrm{I}-\$ 75,000$ & 140 & 13.6 \\
\hline$\$ 75,00 \mathrm{I}-\$ 100,000$ & 231 & 22.4 \\
\hline$>\$ 100,000$ & 564 & 54.7 \\
\hline \multicolumn{3}{|l|}{ Marital status } \\
\hline Married & 949 & 92.0 \\
\hline Divorced & 49 & 4.8 \\
\hline Widowed & 9 & 0.9 \\
\hline Separated & 9 & 0.9 \\
\hline Never married & 10 & 1.0 \\
\hline Unmarried couple & 5 & 0.5 \\
\hline Descriptive statistics & Mean (SD) & Range \\
\hline Age in years & $43.56(5.20)$ & $27-62$ \\
\hline BMI & $25.8(5.60)$ & $|5.8|-54.92$ \\
\hline Years of education & $15.43(1.09)$ & $12-16$ \\
\hline SOC_LADDER & $3.88(1.44)$ & $\mathrm{I}-10$ \\
\hline COM_LADDER & $3.99(1.53)$ & $1-10$ \\
\hline
\end{tabular}

parents and their children, correlations between BMI and the variables listed above, and linear regression for predictors of BMI. Analysis was made using SPSS software. Data are presented as mean \pm standard deviation.

\section{Results}

Demographic information describing the parents is shown in Table 1, which includes race/ethnicity, household income, marital status, and age. Of respondents, $95.8 \%$ were white and $54.7 \%$ had annual incomes over $\$ 100,000$, with $77.1 \%$ over $\$ 75,000$. The overwhelming majority $(92 \%)$ was married, and the mean age was 43.56 years. Figure 1 shows the BMI categories for these female parents, the majority of whom were within the normal weight and with only $45.2 \%$ being overweight/ obese.

Table 2 shows a significant association between parent and child weight status. Clearly, the weight category of children was significantly related to that of their parents $\left[\chi^{2}(9)=83.82, p<0.001\right]$. For parents who were underweight, none of their children were overweight or obese. For parents who were obese, however, $40 \%$ of their children were either overweight or obese.

As seen in Fig. 2, our study showed that the percentage of calories from fat was significantly lower in women with normal weight status compared to women who were overweight or obese $(p=0.001)$. This specific analysis was conducted only on a subset of participants (681 parents of children participating in the 2008 surreys).

To determine the factors that influence BMI, we used linear regression with physical activity (total minutes of MVPA), income, social ladder, and weight perception as independent variables (see Table 3). Since BMI and minutes of physical activity were positively skewed,

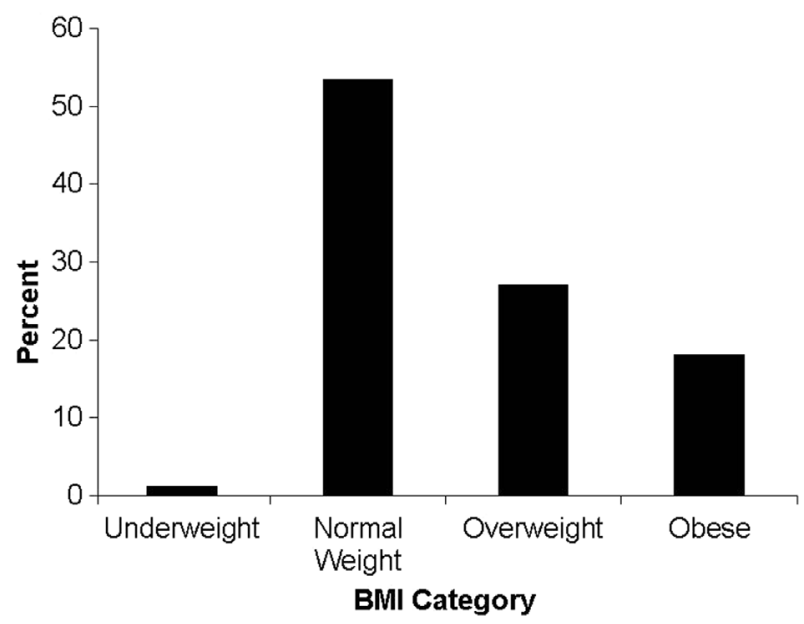

Fig. 1. BMI distribution. A total of 1,032 female parents completed our surveys. BMI distribution in each of the following four categories is indicated: underweight, normal weight, overweight, and obese. 
Table 2. Crosstabulation of parents and children weight status

Children BMI percentile categories

\begin{tabular}{|c|c|c|c|c|c|}
\hline Adult BMI categories & Underweight & Normal weight & Overweight & Obese & Total \\
\hline Underweight count & 2 & 6 & 0 & 0 & 8 \\
\hline$\%$ within BMI categories & 25.0 & 75.0 & 0.0 & 0.0 & 100.0 \\
\hline Normal count & 14 & 366 & 42 & 13 & 435 \\
\hline$\%$ within BMI categories & 3.2 & 84.1 & 9.7 & 3.0 & 100.0 \\
\hline Overweight count & 2 & 152 & 35 & 21 & 210 \\
\hline$\%$ within BMI categories & 1.0 & 72.4 & 16.7 & 10.0 & 100.0 \\
\hline Obese count & 0 & 83 & 26 & 27 & 136 \\
\hline$\%$ within BMI categories & 0.0 & 61.0 & 19.1 & 19.9 & 100.0 \\
\hline Total count & 18 & 607 & 103 & 61 & 789 \\
\hline$\%$ within BMI categories & 2.3 & 76.9 & 13.1 & 7.7 & 100.0 \\
\hline
\end{tabular}

we took the natural logarithms of each. All factors were significant and explained $65 \%$ of BMI's variance $[\mathrm{F}(3,953)=352.52, \quad p<0.001]$. The community ladder was included in an initial model but was not significant. Model residuals and regression diagnostics were examined to assure that assumptions were met.

We also used Pearson correlations to study the impact of each of the above variables one by one (Table 4). As expected, log BMI correlated negatively with physical activity $(-0.25)$, education $(-0.16)$, and income $(-0.25)$. Since a small value on the social scales indicated a high score, $\log$ BMI was positively correlated with social (0.27) and even community (0.18). Self-perception of weight provided the highest correlation (0.79), indicating that the subjects had an accurate perception of their weight. Given our large sample size, most of these correlations were significant ( 0.01 or 0.05 , two-tailed), including for the community ladder, which did not add significantly to the overall linear regression (Table 3).

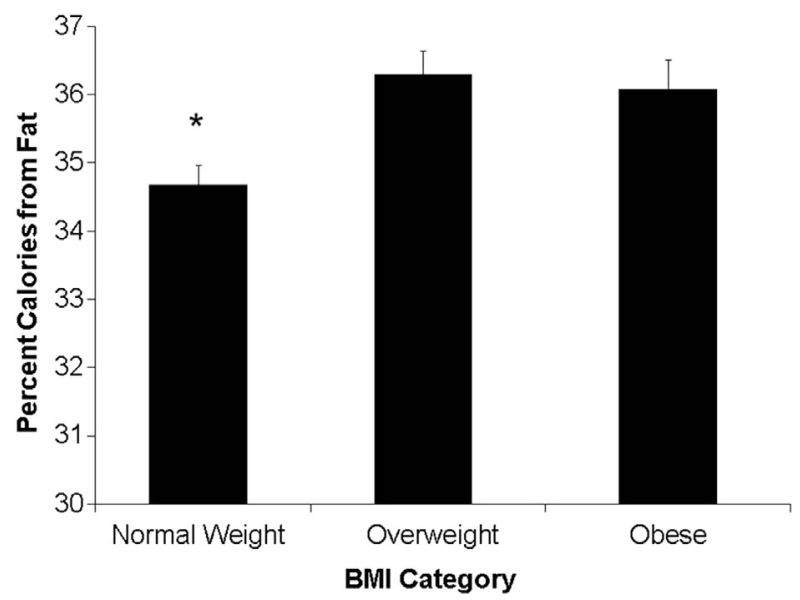

Fig. 2. Fat intake. A validated rapid screener was used to assess fat intake in a subset of the participants $(n=681)$; $* p<0.05$.

\section{Discussion}

In this study, we tested the hypothesized that parents of children who exhibit healthy behaviors and lower BMIs than the average US pediatric population are characterized by lower rates of obesity, higher physical activity, and lower energy intakes.

\section{$B M I$, self-esteem, and SES}

Of female parent participants, 53.5\% were normal weight, $27.1 \%$ were overweight, and $18.1 \%$ were obese (Fig. 1). For comparison, self-reported data from the 2007 BRFSS indicate that only $44.3 \%$ of female respondents were normal weight, $29.8 \%$ were overweight, and $25.6 \%$ were obese (16). Thus, our sample seems to have lower rates of overweight and obesity than the national sample. Among multiple variables assessed, the strongest predictors of BMI were income, fat intake, and time spent in moderate and vigorous physical activity.

In a substudy of the Bogalusa Heart Study that examined the relationship between diet, SES, and demographic factors, adults with higher incomes had healthier diets than adults in the study with lower incomes. Similar results were shown for educational levels. These findings are important, as strong evidence exists suggesting that diet quality significantly impacts weight status (3). Our population exhibited high SES and education level. We believe that the differences in BMI found in our study are not related to these factors, however, as we compared a homogenous sample of participants with similar sex (female), income, education, and SES.

Those females who were the most physically active (MVPA) self-ranked highest in the MacArthur Scale of Subjective Social Status. Among multiple variables assessed, the strongest predictors of BMI were income, fat intake, and time spent in vigorous physical activity. Interestingly, self-reported BMI correlated strongly with how these females described and perceived their weight, which is not usually the case according to the trend 
Table 3. Influence of BMI by physical activity, income, social ladder, education, and weight perception as independent variables

\begin{tabular}{|c|c|c|c|c|c|}
\hline & \multicolumn{3}{|c|}{ Coefficients $^{\mathrm{a}}$} & \multirow[b]{3}{*}{$\mathrm{t}$} & \multirow[b]{3}{*}{ Sig. } \\
\hline & \multicolumn{2}{|c|}{ Unstandardized coefficients } & \multirow{2}{*}{$\frac{\text { Standardized coefficients }}{\text { Beta }}$} & & \\
\hline & B & Std. error & & & \\
\hline (Constant) & 2.811 & 0.073 & & 38.620 & 0.000 \\
\hline PA_TOTAL_In & -0.022 & 0.004 & -0.101 & -5.105 & 0.000 \\
\hline Income & -0.015 & 0.004 & -0.081 & -3.446 & 0.001 \\
\hline SOC_LADDER & 0.008 & 0.003 & 0.059 & 2.494 & 0.013 \\
\hline Years of education & -0.009 & 0.004 & -0.049 & -2.261 & 0.024 \\
\hline How do you describe your weight? & 0.195 & 0.005 & 0.741 & 37.355 & 0.000 \\
\hline
\end{tabular}

âDependent variable: BMI_In.

of underestimating weight and BMI by adults as documented by Gorber et al. (18).

\section{BMI and fat intake}

The females who were within the normal weight category in our study had significantly lower percentage energy intake from fat compared to those who were overweight or obese. Indeed, other studies, including one by Miller et al., compared food consumption of lean adults to that of obese adults and found that leaner women consumed less energy from fat and more fiber compared to obese women. Further, the study found that obese adults consumed more energy from sugar-added foods compared to their lean counterparts (19). Nevertheless, fat intake in our study population was consistent with reported average fat intakes in the United States (20).

Similar findings were shown by a 12-year longitudinal study of diet macronutrient consumption and its association with overweight development of 737 non-overweight women. The study reported a $29 \%$ risk of developing overweight for the subjects consuming high fat diets, while women consuming more fruit, vegetables, and low-fat dairy and meat had overall lower risks, when compared to other dietary groups in this study $(21,22)$. Thus far, research evidence supports weight and health benefits from consuming diets, which are rich in fiber, fruit, and vegetables and low in fat. This recommendation also is supported by major professional and scientific associations.

\section{Physical activity and obesity}

In our study, the mean MVPA of female parents was $360 \mathrm{~min} /$ week. According to NHANES 2005-2006 data, only $55.8 \%$ of adult females in the United States meet the recommended physical activity guidelines (23). In contrast, $76.5 \%$ of female parents in our study met these requirements. Thus, the findings about the physical activity of the mothers surveyed in our study are consistent with their lower BMIs. Indeed, previous studies have shown that a higher fitness level is associated with leisuretime physical activity in young to middle-aged adults (24). Overall, research strongly suggests that women following the physical activity recommendations had significantly lower BMIs and waist-to-hip ratios and improved cardiorespiratory fitness (10).

\section{Parental influence on children's BMI}

We have previously shown that children participating in a creative afterschool program were more physically active (compared to national averages in meeting recommendations) and exhibited overall lower BMI rates, lower screen times, and higher intake of fruits, vegetables, and milk (25). In the current study, we found that the female parents were highly physically active (based on recommended amounts of MVPA). Although we previously reported that their children also were physically active, a direct cause-effect cannot be established within this study. BMI of children and their parents were closely associated, as the prevalence of obesity and overweight in children was higher when parents also were overweight or obese (Table 2), indicating close association between parent and children weight status; this relationship could be due to both genetic and environmental influences. Given the high physical activity of this parent population, however, it is possible that the family lifestyle influenced children's weight status. These results also are consistent with previous reports showing associations between parents' BMI and that of their children (26).

\section{Conclusions}

We report here results obtained for the female parents. Interestingly, only $45 \%$ of these female parents were overweight/obese, compared to a national average of $64.1 \%$ for females. Most parents $(76 \%)$ had completed a college degree and earned over $\$ 75,000$ a year. Parents who reported the lowest income were the most obese in this population. Among multiple variables assessed, the strongest predictors of BMI were income, fat intake, and 
Table 4. Pearson correlations (two-tailed for various variables)

\begin{tabular}{|c|c|c|c|c|c|c|c|}
\hline & \multicolumn{7}{|c|}{ Correlations } \\
\hline & BMI_ln & PA_TOT_In & Income & SOC_LAD-DER & COM_LAD-DER & Education & $\begin{array}{l}\text { Weight } \\
\text { perception }\end{array}$ \\
\hline \multicolumn{8}{|l|}{ BMI_In } \\
\hline Pearson correlation & I & $-0.254 * *$ & $-0.246^{* *}$ & $0.267^{* *}$ & $0.181 * *$ & $-0.164 * *$ & $0.788 * *$ \\
\hline Sig. (two-tailed) & & 0.000 & 0.000 & 0.000 & 0.000 & 0.000 & 0.000 \\
\hline$N$ & 1,017 & 951 & 1,017 & 1,017 & 1,015 & 1,016 & 1,016 \\
\hline \multicolumn{8}{|l|}{ PA_TOT_In } \\
\hline Pearson correlation & $-0.254^{* *}$ & 1 & $0.077^{*}$ & $-0.132 * *$ & $-0.104 * *$ & 0.006 & $-0.186 * *$ \\
\hline Sig. (two-tailed) & 0.000 & & 0.017 & 0.000 & 0.001 & 0.860 & 0.000 \\
\hline$N$ & 951 & 964 & 963 & 964 & 963 & 961 & 963 \\
\hline \multicolumn{8}{|l|}{ Income } \\
\hline Pearson correlation & $-0.246 * *$ & $0.077^{*}$ & I & $-0.54 I^{* *}$ & $-0.292^{* *}$ & $0.399 * *$ & $-0.148^{* *}$ \\
\hline Sig. (two-tailed) & 0.000 & 0.017 & & 0.000 & 0.000 & 0.000 & 0.000 \\
\hline$N$ & 1,017 & 9,63 & $|, 03|$ & $|, 03|$ & 1,029 & 1,029 & 1,030 \\
\hline \multicolumn{8}{|l|}{ SOC_LADDER } \\
\hline Pearson correlation & $0.267^{* *}$ & $-0.132^{* *}$ & $-0.54 I^{* *}$ & 1 & $0.614 * *$ & $-0.410 * *$ & $0.188 * *$ \\
\hline Sig. (two-tailed) & 0.000 & 0.000 & 0.000 & & 0.000 & 0.000 & 0.000 \\
\hline$N$ & $\mathrm{I}, 017$ & 964 & $|, 03|$ & 1,032 & 1,030 & 1,029 & 1,031 \\
\hline \multicolumn{8}{|l|}{ COM_LADDER } \\
\hline Pearson correlation & $0.181 * *$ & $-0.104 * *$ & $-0.292 * *$ & $0.614 * *$ & I & $-0.245 * *$ & $0.135 * *$ \\
\hline Sig. (two-tailed) & 0.000 & 0.001 & 0.000 & 0.000 & & 0.000 & 0.000 \\
\hline$N$ & 1,015 & 963 & 1,029 & 1,030 & 1,030 & $\mathrm{I}, 027$ & 1,029 \\
\hline \multicolumn{8}{|l|}{ Education } \\
\hline Pearson correlation & $-0.164 * *$ & 0.006 & $0.399 * *$ & $-0.410^{* *}$ & $-0.245^{* *}$ & I & $-0.078^{*}$ \\
\hline Sig. (two-tailed) & 0.000 & 0.860 & 0.000 & 0.000 & 0.000 & & 0.013 \\
\hline$N$ & 1,016 & 961 & 1,029 & 1,029 & $\mathrm{I}, 027$ & 1,029 & $\mathrm{I}, 028$ \\
\hline \multicolumn{8}{|l|}{ Weight perception } \\
\hline Pearson correlation & $0.788 * *$ & $-0.186 * *$ & $-0.148 * *$ & $0.188^{* *}$ & $0.135 * *$ & $-0.078 *$ & 1 \\
\hline Sig. (two-tailed) & 0.000 & 0.000 & 0.000 & 0.000 & 0.000 & 0.013 & \\
\hline$N$ & 1,016 & 963 & $\mathrm{I}, 030$ & $\mathrm{I}, 03 \mathrm{I}$ & 1,029 & $\mathrm{I}, 028$ & $|, 03|$ \\
\hline
\end{tabular}

*Correlation is significant at the 0.05 level (two-tailed).

**Correlation is significant at the 0.01 level (two-tailed).

time spent in vigorous physical activity. Those females who were the most physically active self-ranked highest in the MacArthur Scale of Subjective Social Status. Interestingly, self-reported BMI correlated strongly with how these females described and perceived their weight, which is not usually the case.

These studies demonstrate that female parents of children participating in an afterschool creative program were physically active, which likely accounts for the parents' lower overweight/obesity rates. We found that this group of mothers exhibited much healthier weight status and lifestyle behaviors compared to their counterparts in the general population. These findings further support the importance of parent modeling
(6) in the management of overweight and obesity in children.

\section{Limitations}

Race was not considered in our study, as the minority sample was small (4.2\%). According to Flegal et al., however, significant differences exist in women of various minority backgrounds, compared to the overall overweight and obesity prevalence among women in the United States (64.1\% in 2008). In non-Hispanic white women, obesity prevalence was $33 \%$, whereas obesity was found to be at $49.6 \%$ among non-Hispanic black women. Further, the study found that, in comparison to nonHispanic white women, non-Hispanic black and Mexican- 
American women were more significantly more likely to be obese (odds ratio 2.26 and 2.51, respectively). Approximately $7 \%$ of US women are classified to be at the grade 3 level of obesity (BMI $\geq 40$ ), with this obesity level more commonly found among non-Hispanic black women $(14.2 \%)(2)$.

Our population was primarily Caucasian, which is important to take into consideration as it is well established that differences in overweight and obesity prevalence exist among women of various ethnic and race backgrounds (2). Evidence shows that obesity and lifestyle practices vary by SES (3). Given our population's higher income and education status and low proportion of minorities, our findings cannot be directly translated across all ethnic backgrounds and education/socioeconomic levels. Thus, further studies are warranted in minority populations.

Participating parents were predominantly mothers from which data reported here were collected. Fewer responses were obtained from male parents/guardians, so males were excluded in our methods. Interestingly, unlike our observed low rates of overweight and obesity in the female parents, male parents exhibited comparable rates to the average BMIs rates for the country. The number of fathers responding ( $n=156$ for 2008 and 2009 surveys) may not be a statistically valid sample, so future studies should consider the difference between male and female parents of fit children, including whether fathers are education oriented.

Review of the literature strongly supports the role of parent/adult modeling and styles in influencing physical activity and dietary intakes. Thus, more public health strategies are needed to tackle the obesity epidemic, especially in relation to parental influence on children's health-related behavior. Future studies also should consider (1) parents of child populations who are not involved in a program such as DI and (2) fit children from diverse backgrounds.

\section{Acknowledgments}

This project was supported by National Research Initiative Grant 2008 55215-18799 from the USDA National Institute for Food and Agriculture. We thank the children and parents who participated in the study, Robert Gibbs for facilitating communication with DI, and Carolyn Berryhill for her technical assistance in preparing and editing this manuscript. We also thank the DI staff for facilitating this study and providing access to their members and parents.

\section{Conflict of interest and funding}

The authors declare that they have no competing interests.

\section{Authors' contributions}

NMM and CC designed this original study and secured its funding. NMM, CC, BG, EF, MS, and NSK approved the research design and participated in data collection and analysis. RM assisted with statistical analyses. All authors read and approved the final manuscript.

\section{References}

1. Bray MS. Implications of gene-behavior interactions: prevention and intervention for obesity. Obesity 2008; 16(Suppl 3): S72-8.

2. Flegal KM, Carroll MD, Ogden CL, Curtin LR. Prevalence and trends in obesity among US adults, 1999-2008. JAMA 2010; 303(3): 235-41.

3. Desmukh-Taskar P, Nicklas TA, Yang SJ, Berenson GS. Does food group consumption vary by differences in socioeconomic, demographic, and lifestyle factors in young adults? The Bogalusa heart study. JADA 2007; 107(2): 223-34.

4. Overweight and obesity; http://www.cdc.gov/obesity/causes/ health.html

5. Childhood overweight and obesity; http://www.cdc.gov/obesity/ childhood/index.html

6. Savage JS, Fisher JO, Birch LL. Parental influence on eating behavior: conception to adolescence. J Law Med Ethics 2007; 35(1): 22-34

7. Obesity and overweight 2009; http://www.who.int/mediacentre/ factsheets/fs311/en/index.html

8. Physical activity guidelines for Americans 2008; http://www. health.gov/paguidelines/factsheetprof.aspx

9. Jakicic JM, Marcus BH, Gallagher KI, Napolitano M, Lang W. Effect of excercise duration and intensity on weight loss in overweight, sedantary women: a randomized trial. JAMA 2003; 290(10): 1323-30.

10. Holcomb CA, Heim DL, Loughin TM. Physical activity minimizes the association of body fatness with abdominal obesity in white premenopausal women: results from the third National Health and Nutrition Examination. JADA 2004; 104 : 1859-62.

11. Davis CL, Cooper S. Fitness, fatness, cognition, behavior, and academic achievement among overweight children: do crosssectional associations correspond to exercise trial outcomes? Prev Med 2011; 52(Suppl 1): S65-9.

12. Fuemmeler BF, Anderson CB, Masse LC. Parent-child relationship of directly measured physical activity. Int J Behav Nutr Phys Act 2011; 8(1): 17.

13. Hennessy E, Hughes SO, Goldberg JP, Hyatt RR, Economos CD. Parent-child interactions and objectively measured child physical activity: a cross-sectional study. Int J Behav Nutr Phys 2010; 7: 71.

14. Jago R, Fox KR, Page AS, Brockman R, Thompson JL. Parent and child physical activity and sedentary time: do active parents foster active children? BMC Public Health 2010; 10: 194.

15. Hills AP, Okely AD, Baur LA. Addressing childhood obesity through increased physical activity. Nat Rev Endocrinol 2010; 6(10): 543-9.

16. Behavioral Risk Factor Surveillance System 2007; www.cdc.gov/ brfss/questionnaires/pdf-ques/2007brfss.pdf

17. Block G, Gillespie C, Rosenbaum EH, Jenson C. A rapid food screener to assess fat and fruit and vegetable intake. Am J Prev Med 2000; 18: 284-8.

18. Gorber SC, Tremblay M, Moher D, Gorber B. A comparison of direct vs. self-report measures for assessing height, weight and body mass index: a systematic review. Obes Rev 2007; 8(4): 307-36.

19. Miller WC, Neiderpruem MG, Wallace JP, Lindeman AK. Dietary fat, sugar, and fiber predict fat content. JADA 1994; 94(6): $612-5$. 
20. Wright JD, Wang CY. Trends in intake of energy and macronutrients in adults from 1999-2000 through 2007-2008. U.S. Department of Health and Human Services. Centers for Disease Control and Prevention National Center for Health Statistics. NCHS Data Brief No. 49; November 2010.

21. Quatromani PA, Copenhafer DL, D'Agostino RB, Millen BE. Dietary patterns predict the development of overweight in women: the Framingham Nutrition Studies. JADA 2002; 102(9): $1240-6$.

22. Howarth NC, Huang TTK, Roberts SB, McCory MA. Dietary fiber and fat are associated with excess weight in young and middle-aged US adults. JADA 2005; 105(9): 1365-72.

23. Tucker JM, Welk GJ, Beyler NK. Physical activity in U.S. adults compliance with the physical activity guidelines for Americans. Am J Prev Med 2011; 40(4): 454-61.

24. Wang CY, Haskell WL, Farrell SW, Lamonte MJ, Blair SN, Curtin LR, et al. Cardiorespiratory fitness levels among US adults 20-49 years of age: findings from the 1999-2004 National
Health and Nutrition Examination Survey. Am J Epidemiol 2010; 171(4): 426-35.

25. Costello C, Greer B, Fitzhugh E, Spence M, Muenchen R, Moustaid-Moussa N. BMI, perceived weight status, physical activity, and dietary consumption between middle and high school students. Obesity 2020; 18(Suppl 2): S37.

26. Whitaker RC, Wright JA, Pepe MS, Seidel KD, Dietz WH. Predicting obesity in young adulthood from childhood and parental obesity. N Eng J Med 1997; 337(13): 869-73.

*Naima Moustaid-Moussa

Texas Tech University

College of Human Sciences

Nutritional Sciences program

I30I Akron Avenue

Lubbock, TX 79409, USA

Email: naima.moustaid-moussa@ttu.edu 